\title{
Hopf Bifurcation of Compound Stochastic van der Pol System
}

\author{
Shaojuan Ma and Qianling Zhang \\ School of Mathematics \& Information Science, Beifang University of Nationalities, Yinchuan 750021, China \\ Correspondence should be addressed to Shaojuan Ma; dr.sjma@gmail.com
}

Received 9 December 2015; Revised 5 May 2016; Accepted 16 May 2016

Academic Editor: Haranath Kar

Copyright (C) 2016 S. Ma and Q. Zhang. This is an open access article distributed under the Creative Commons Attribution License, which permits unrestricted use, distribution, and reproduction in any medium, provided the original work is properly cited.

\begin{abstract}
Hopf bifurcation analysis for compound stochastic van der Pol system with a bound random parameter and Gaussian white noise is investigated in this paper. By the Karhunen-Loeve (K-L) expansion and the orthogonal polynomial approximation, the equivalent deterministic van der Pol system can be deduced. Based on the bifurcation theory of nonlinear deterministic system, the critical value of bifurcation parameter is obtained and the influence of random strength $\delta$ and noise intensity $\sigma$ on stochastic Hopf bifurcation in compound stochastic system is discussed. At last we found that increased $\delta$ can relocate the critical value of bifurcation parameter forward while increased $\sigma$ makes it backward and the influence of $\delta$ is more sensitive than $\sigma$. The results are verified by numerical simulations.
\end{abstract}

\section{Introduction}

In recent years, the research of dynamics behaviors have been extensively concentrated on the stochastic nonlinear dynamical systems, whose internal uncertainty of system forming random parameter and external actions of system forming random excitation are modeled as different noise processes.

There are some results about these different kinds of stochastic systems. The first kind of stochastic dynamical systems are the systems driven by stochastic parameter excitation or external noise excitation idealized as Gaussian white noise processes. Dynamics behaviors for stochastic van der Pol system and its coupled systems with parametric and external forces have been investigated by the stochastic averaging method [1-6], such as response analysis, Hopf bifurcation, and chaos. The existence of Hopf bifurcation about stochastic van der Pol system treating driving term as a randomly fluctuating quantity is obtained $[7,8]$. For the nonlinear system with external Gaussian white noise, various properties of the response are presented [9-11]. With the cumulant-neglect closure method, the statistical moments in a Duffing system responding to random excitation are established [12]. The possibility for onset of chaotic motion in the Duffing oscillator under parameter excitation of bound noise is studied by the stochastic Melnikov method [13]. The second kind of stochastic systems are the systems with only internal random parameter considered as time-invariant and that served as random fields or as random variables. The orthogonal series expansion using a vector of random variables to represent the random field is widely exploited and applied in the stochastic structural systems [14-17]. Li and Fang et al. [18-21] have developed the orthogonal polynomial method to approximate the dynamics response of linear random structure by a series of orthogonal basis functions. Owing to the representation of random parameters as random fields greatly increases the size and complexity of the analysis, Ma et al. [22-25] have applied the method of orthogonal polynomial approximation to study various dynamics behaviors of nonlinear systems with random parameter idealized as random variables and have verified this method as feasible and effective.

However, there also exist other stochastic systems with both random fields and external noise processes. By transforming from stochastic Duffing system to the deterministic structural Duffing system with external noise excitation, [26] has obtained the statistical moments and the probability density function of response to study the reliability analysis of structure. The hybrid polynomial chaos and pseudoexcitation method have been proposed to evaluate the random vibration response in the linear structure system with uncertain parameters under external random excitation [27]. 
With the combination of the orthogonal polynomial approximation and the K-L expansion, the sequential orthogonal decomposition is developed to discuss the response analysis of ground motion by $\operatorname{Li}[28,29]$. The precise integration method is used to obtain the K-L decomposition of the nonstationary filtered white noise random excitation; then based on the linear superposition principle, each element of the K-L vector is added to each equation of the extended order system by the form of one to one and a small amount of $\mathrm{K}-\mathrm{L}$ vector used to compute response of the extended order system can obtain a good result [30].

In this paper, we name the stochastic system with both random parameter treating as random variables and external noise processes as compound (or double) stochastic dynamical system. For random variables conforming to arch-like probability density function, a bound random parameter van der Pol system with external Gaussian white noise is considered, and the response of the system is presented by two sets of orthogonal polynomial bases in random function space under the condition of convergence in mean square. Compared with $[26,27,30]$, the random response of this paper for the stochastic van der Pol system is directly expressed as the double series form of deterministic response using the known orthogonal polynomial functions by means of the sequential orthogonal decomposition. So this paper can be viewed as an extensive and supplementary version of the related literature. On the other hand, there are a few results to explore the stochastic bifurcation in this kind of stochastic system. So the stochastic Hopf bifurcation of van der Pol system with a bound random parameter and Gaussian white noise is explored in detail in this paper.

This paper is organized as follows. In Section 2, a new van der Pol system with a bound random parameter and Gaussian white noise is simplified into its equivalent deterministic one. The critical value of Hopf bifurcation parameter is given by the bifurcation theory of deterministic system and the effect of random strength and noise intensity on the critical value of bifurcation parameter is analyzed in Section 3. In the same section the results are verified by numerical simulations. Section 4 concludes the influence of random parameter and Gaussian white noise on Hopf bifurcation in stochastic van der Pol system.

\section{The Equivalent Deterministic One for Compound Stochastic van der Pol System}

Excited by internal random parameter and Gaussian white noise, compound stochastic van der Pol system is established in this paper:

$$
\ddot{x}+\left(\alpha x^{2}-\bar{\beta}\right) \dot{x}+x=\sigma F(t)
$$

where $\alpha$ and $\bar{\beta}$ are deterministic parameter and random parameter, respectively. $F(t)$ is Gaussian white noise with zero mean and its intensity is $\sigma$.
Random parameter $\bar{\beta}$ can be defined as

$$
\bar{\beta}=\beta+\delta u
$$

where $u$ is random variable obeying arch-like probability density function defined on $[-1,1]$ and $\beta$ and $\delta$ are all the statistical parameters of $\bar{\beta}$.

Using eigenfunctions as the orthogonal bases, stochastic noise process $F(t)$ can be expressed as the denumerable series form called the K-L expansion [31-33], so the K-L expansion of $F(t)$ is

$$
F(t)=\sum_{n=0}^{N} \xi_{n} \sqrt{\lambda_{n}} f_{n}(t),
$$

where $\lambda_{n}$ and $f_{n}(t)$ are the eigenvalues and eigenfunctions of the covariance function of $F(t)$, and eigenfunctions $f_{n}(t)$ form a set of complete orthogonal bases in complete Hilbert space and satisfy $\int_{0}^{\infty} f_{n}(t) f_{m}(t) d t=\delta_{n m}$, in which $\delta_{n m}$ is the Kronecker function. $\xi_{n}$ are independent orthogonal random variables obeying normal distribution and have the properties of $E\left(\xi_{n}\right)=0, E\left(\xi_{n}^{2}\right)=1$.

Eigenvalues and eigenfunctions of the K-L expansion for Gaussian white noise with zero mean are given [34], namely, $\lambda_{n}=1 /\left[(n+1 / 2)^{2} \pi^{2}\right]$ and $f_{n}(t)=\sqrt{2}((n+1 / 2) \pi t)$.

The only fixed point of system (1) is $A(\sigma F(t), 0)$ when $\sigma \neq 0$ and can be further transformed into the original point $\mathrm{O}(0,0)$. Through the coordinate transformation, system (1) is rewritten as coupled first-order system (here is still noted by $x)$ :

$$
\begin{aligned}
& \dot{x}=y, \\
& \dot{y}=-x+\left(\bar{\beta}-\alpha \sigma^{2} F^{2}(t)\right) y-\alpha(x+2 \sigma F(t)) x y .
\end{aligned}
$$

By the sequential orthogonal decomposition, the response of system (4) can be expressed in the following double series form:

$$
\begin{aligned}
& x(\xi, u, t)=\sum_{i=0}^{N} \sum_{j=0}^{M} H_{i}(\xi) T_{j}(u) x_{i j}(t), \\
& y(\xi, u, t)=\sum_{i=0}^{N} \sum_{j=0}^{M} H_{i}(\xi) T_{j}(u) y_{i j}(t),
\end{aligned}
$$

where $x_{i j}(t)=\int_{\Omega_{\xi}} \int_{\Omega_{u}} x(\xi, u, t) H_{i}(\xi) T_{j}(u) \omega(\xi) \theta(u) d \xi d u$ and $y_{i j}(t)=\int_{\Omega_{\xi}} \int_{\Omega_{u}} y(\xi, u, t) H_{i}(\xi) T_{j}(u) \omega(\xi) \theta(u) d \xi d u$, in which $\omega(\xi)$ and $\theta(u)$ are normal probability density function and arch-like probability density function, respectively. Corresponding to these probability density functions, Hermite orthogonal polynomial $H_{i}(\xi)$ and Chebyshev orthogonal polynomial of the second kind $T_{j}(u)$ can be taken as orthogonal bases. $N$ and $M$ are the truncation order of the series we have taken. In addition, it is assumed that random variables $\xi$ and $u$ are mutually independent. 
Substituting (2), (3), and (5) into (4), equivalent deterministic van der Pol system can be given when $N \rightarrow \infty$ and $M \rightarrow \infty$. So as to make the following numerical simulations effective and convenient, we take $N=M=1$ and approximately obtain the equivalent deterministic system of compound stochastic van der Pol system:

$$
\begin{aligned}
\dot{x}_{00}(t)= & y_{00}(t), \\
\dot{y}_{00}(t)= & -x_{00}(t)+\beta y_{00}(t)+\frac{\delta}{2} y_{01}(t)-\alpha M_{00}(t) \\
& -\alpha \sigma^{2}\left(\lambda_{0}+\lambda_{1}\right) y_{00}(t), \\
\dot{x}_{01}(t)= & y_{01}(t), \\
\dot{y}_{01}(t)= & -x_{01}(t)+\beta y_{01}(t)+\frac{\delta}{2} y_{00}(t)-\alpha M_{01}(t) \\
& -\alpha \sigma^{2}\left(\lambda_{0}+\lambda_{1}\right) y_{01}(t), \\
\dot{x}_{10}(t)= & y_{10}(t), \\
\dot{y}_{10}(t)= & -x_{10}(t)+\beta y_{10}(t)+\frac{\delta}{2} y_{11}(t)-\alpha M_{10}(t) \\
& -\alpha \sigma^{2}\left(\lambda_{0}+\lambda_{1}\right) y_{10}(t), \\
& -\alpha \sigma^{2}\left(\lambda_{0}+\lambda_{1}\right) y_{11}(t), \\
\dot{x}_{11}(t)= & y_{11}(t), \\
\dot{y}_{11}(t)= & -x_{11}(t)+\beta y_{11}(t)+\frac{\delta}{2} y_{10}(t)-\alpha M_{11}(t)
\end{aligned}
$$

where $M_{i j}(t)(i=0,1, j=0,1)$ notes the coefficient of $H_{i}(\xi) T_{j}(u)$ in the linear combination.

The deterministic parameter for system (6) can be taken as $\alpha=1.0$ and initial values are

$$
\begin{aligned}
x_{00}(0) & =0.3, \\
y_{00}(0) & =0.2, \\
x_{i j}(t) & =y_{i j}(t)=0 \quad(i=0,1, j=0,1) .
\end{aligned}
$$

\section{Bifurcation Parameter and Numerical Simulations}

According to the Hopf bifurcation theory of deterministic nonlinear system $[35,36]$, the critical value of bifurcation parameter for the equivalent deterministic system (6) is obtained as follows:

$$
\beta_{c}=\frac{80 \sigma^{2}-9 \pi^{2} \delta}{18 \pi^{2}}
$$

By the following numerical simulations, the influence of random strength $\delta$ and noise intensity $\sigma$ on bifurcation parameter $\beta_{c}$ is discussed in this section. Figure 1 clearly shows the theoretical relationship among $\delta, \sigma$, and $\beta_{c} . \beta_{c}$ decreases as $\delta$ increases while $\beta_{c}$ increases as $\sigma$ increases. In other words, $\delta$ can induce the generation of Hopf bifurcation but $\sigma$ delays it.

System (4) is the deterministic van der Pol system when we take $\delta=\sigma=0$. Meanwhile, the bifurcation parameter $\beta$ equals to $\beta_{c}=0$ and the system shows limit cycles at the fixed point $O(0,0)$. Hopf bifurcation phase diagrams and time history diagrams are plotted in Figure 2 when we, respectively, take $\beta$ less than and greater than $\beta_{c}$.

The stochastic van der Pol system with a bound random parameter is the case of $\delta \neq 0$ and $\sigma=0$. Random variable $u$ continually changes for the random response; we can take its ensemble mean response to replace it. Taking $\delta=0.1$, we obtain $\beta_{c}=-0.05$. When $\beta_{c}>\beta=-0.11$, it takes more time to gradually converge at the fixed point for the trajectory of ensemble mean response than the deterministic system shown in Figures 3(a) and 3(c). The trajectory of ensemble mean response is gradually convergent closed curve when $\beta_{c}<\beta=-0.045$ and time used for converging is shorter than the deterministic one shown in Figures 3(b) and 3(d). Compared with the deterministic van der Pol system, the critical value of bifurcation parameter is smaller and Hopf bifurcation occurred in advance.

It is also stochastic van der Pol system with $\delta=0$ and $\sigma \neq 0$. We take the ensemble mean response about random variable $\xi$ to substitute the random response. Taking $\sigma=0.1$, we obtain $\beta_{c}=0.0045$. When $\beta_{c}>\beta=-0.085$, the trajectory is gradually convergent at the fixed point described in Figures $4(\mathrm{a})$ and $4(\mathrm{c})$. The trajectory is gradually convergent closed curve when $\beta_{c}<\beta=0.005$ also described in Figures 4(b) and $4(\mathrm{~d})$. Compared with the deterministic van der Pol system, the noise intensity $\sigma$ of stochastic van der Pol system makes the critical value of bifurcation parameter larger and Hopf bifurcation occurred later.

The compound stochastic van der Pol system is $\delta \neq 0$ and $\sigma \neq 0$. We still take the ensemble mean response about random variables $u$ and $\xi$ to stand for the random response (5) and give the bifurcation phase diagram and time history diagram when $\delta=\sigma=0.1$ and $\beta<\beta_{c}$ (see Figure 5). Comparing Figure 5(b) with Figure 2(c), it is found that increased $\delta$ postpones time of convergence and promotes the generation of Hopf bifurcation at the fixed point, and increased $\sigma$ shortens time of convergence and postpones the generation of Hopf bifurcation. Meanwhile, it is obvious that the range for the larger value of the ensemble mean response shown in Figure 5(b) is the same as the result shown in Figure 3(c) and is larger than the result described in Figure 4(c).

From the above numerical simulations, it is discovered that $\delta$ and $\sigma$ have the different influence on the critical value of Hopf bifurcation parameter in the compound stochastic van der Pol system. As we increase the random parameter strength $\delta$, the critical value of bifurcation parameter is smaller than the deterministic system. While we increase the noise intensity $\sigma$, the critical value of bifurcation parameter is larger than the deterministic system. Simultaneously, the 


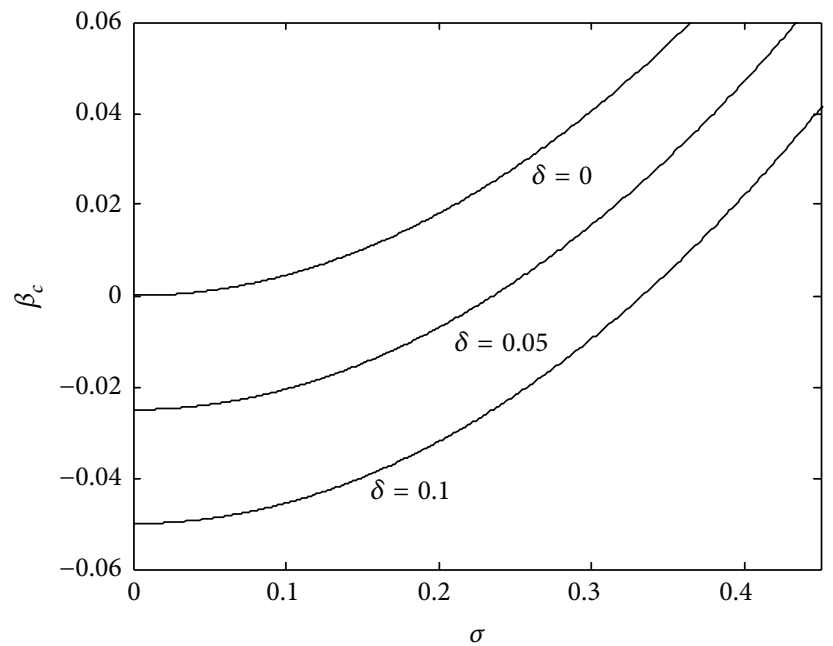

FIGURE 1: The function diagram between the bifurcation parameter and two kinds of random intensity.

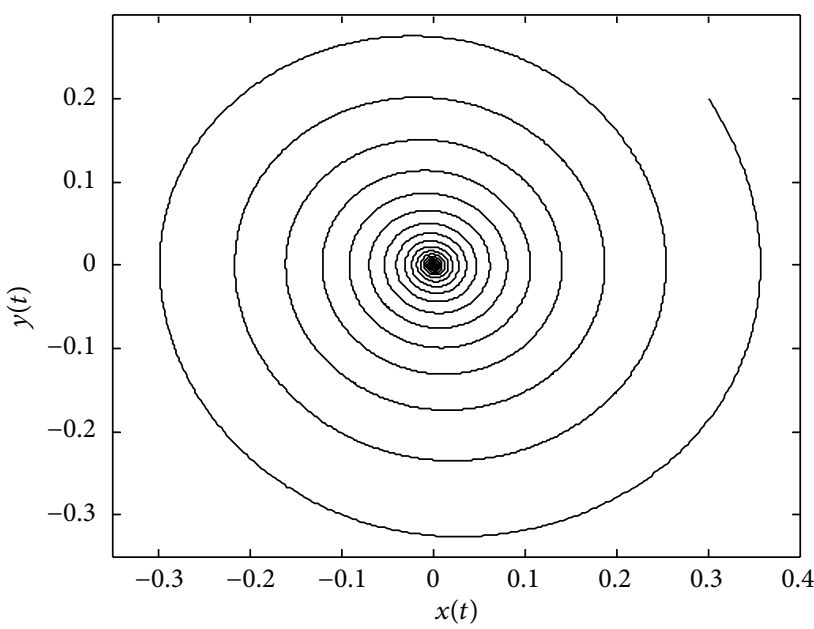

(a) $\beta<\beta_{c}$

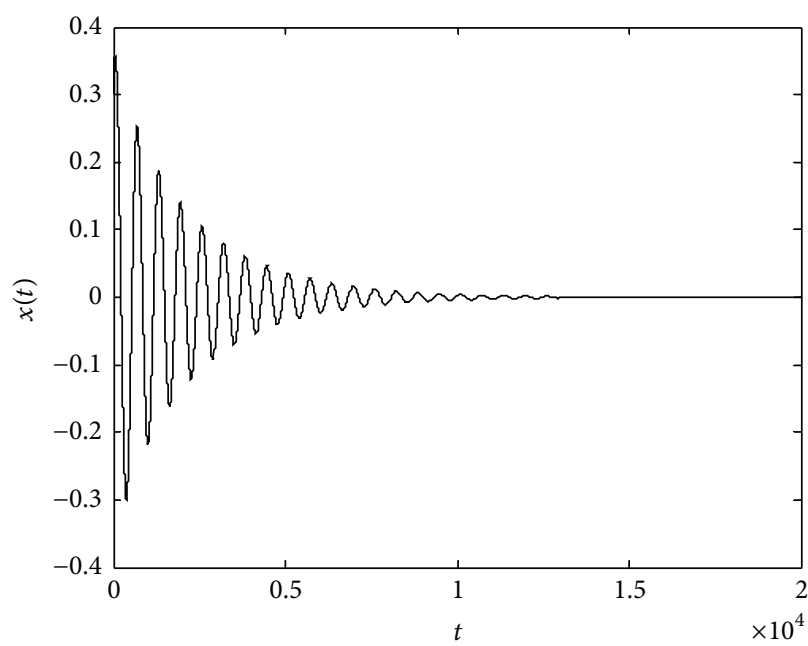

(c) $\beta<\beta_{c}$

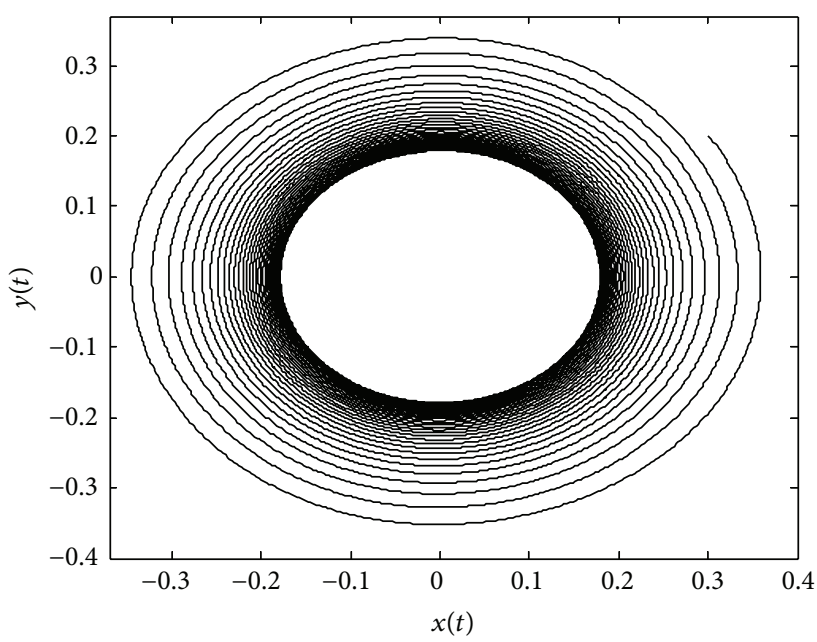

(b) $\beta>\beta_{c}$

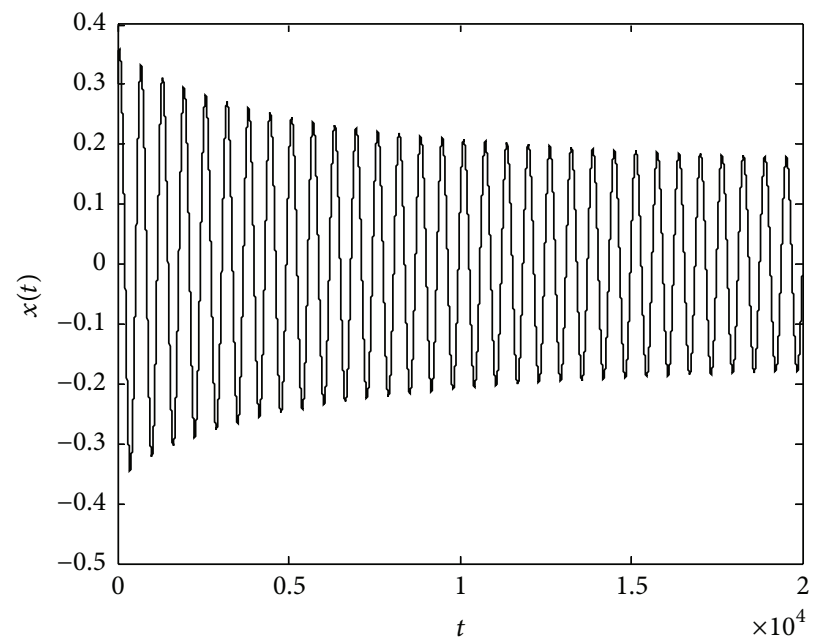

(d) $\beta>\beta_{c}$

FIgUre 2: The Hopf bifurcation phase diagrams and time history diagrams of deterministic van der Pol system. 


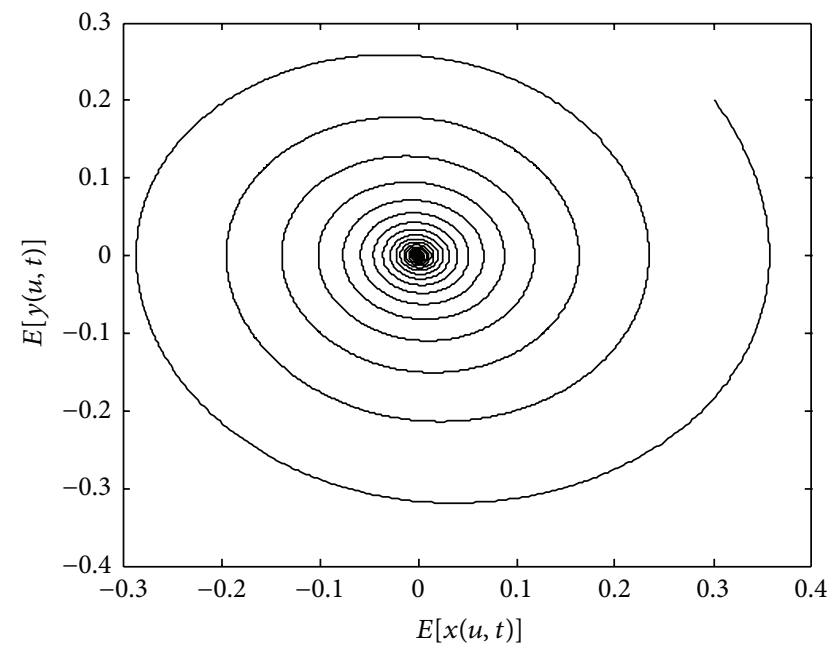

(a) $\delta=0.1, \beta<\beta_{c}$

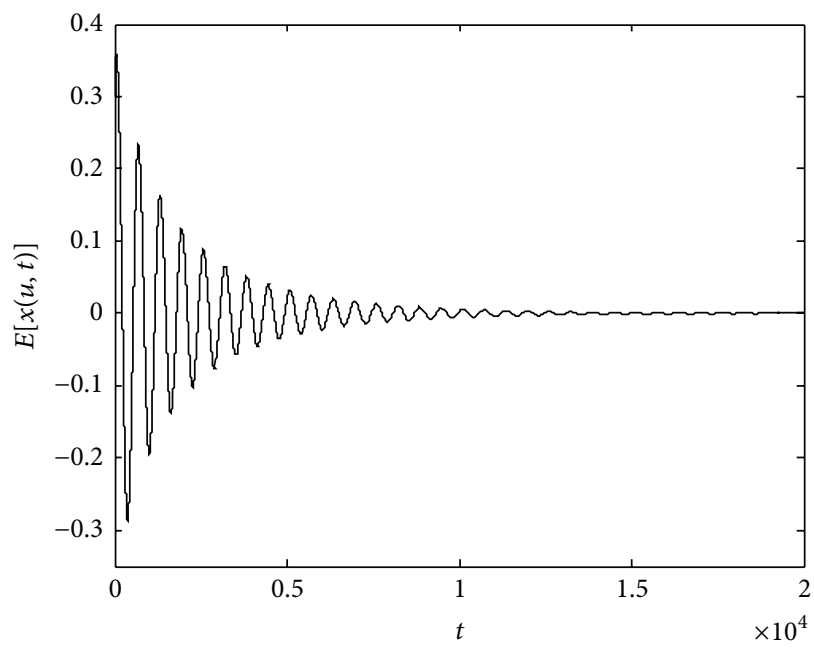

(c) $\delta=0.1, \beta<\beta_{c}$

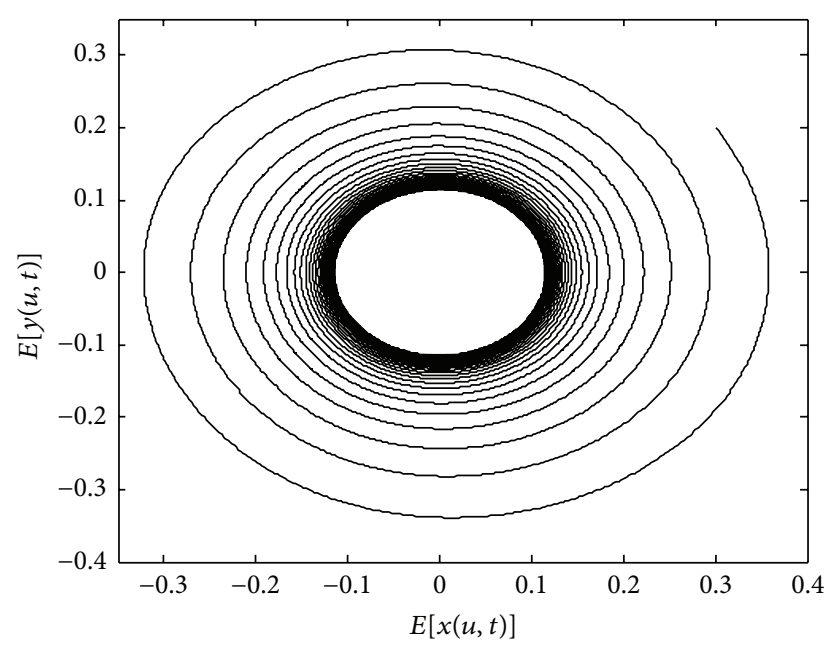

(b) $\delta=0.1, \beta>\beta_{c}$

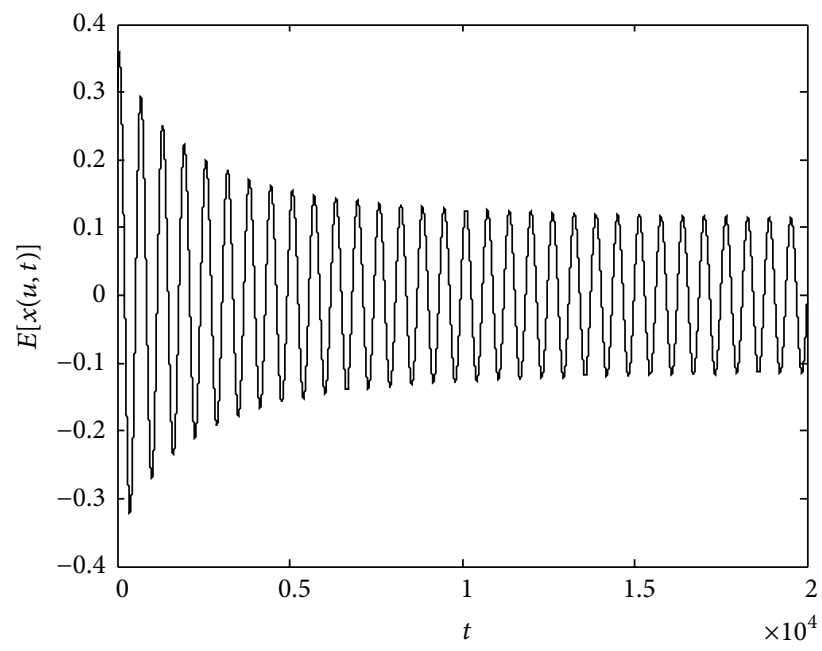

(d) $\delta=0.1, \beta>\beta_{c}$

FIgURE 3: The bifurcation phase diagrams and time history diagrams of a bound random parameter van der Pol system.

influence of $\delta$ on the critical value of bifurcation parameter is more sensitive than $\sigma$ taking the same value.

\section{Conclusion}

In this paper, we deal with Hopf bifurcation of compound stochastic van der Pol system with random parameter and Gaussian white noise. It shows that the method of sequential orthogonal decomposition combining the orthogonal polynomial approximation with the K-L expansion is effective to deduce the stochastic van der Pol system into its equivalent deterministic system; then the theory of Hopf bifurcation in nonlinear deterministic system is applied to the equivalent one and thereby Hopf bifurcation of stochastic van der Pol system under random parameter and Gaussian white noise is analyzed. In addition, it is found that these two kinds of random intensity have the different effects on the critical value of Hopf bifurcation parameter for compound stochastic van der Pol system and the influence of $\delta$ is more outstanding than $\sigma$ in the same value. Finally, we verify the results by numerical simulations.

The compound stochastic van der Pol system established in this paper is also available for other nonlinear stochastic dynamical systems. And various dynamics behaviors in such systems as an extensive and supplementary version of the related literature should have been studied deeply, such as different bifurcations, stability, chaotic motion, and numerical analysis of response. It is especially worth taking into account the convergence study of response prediction because of a series with finite terms.

\section{Competing Interests}

The authors declare that there are no competing interests regarding the publication of this paper. 


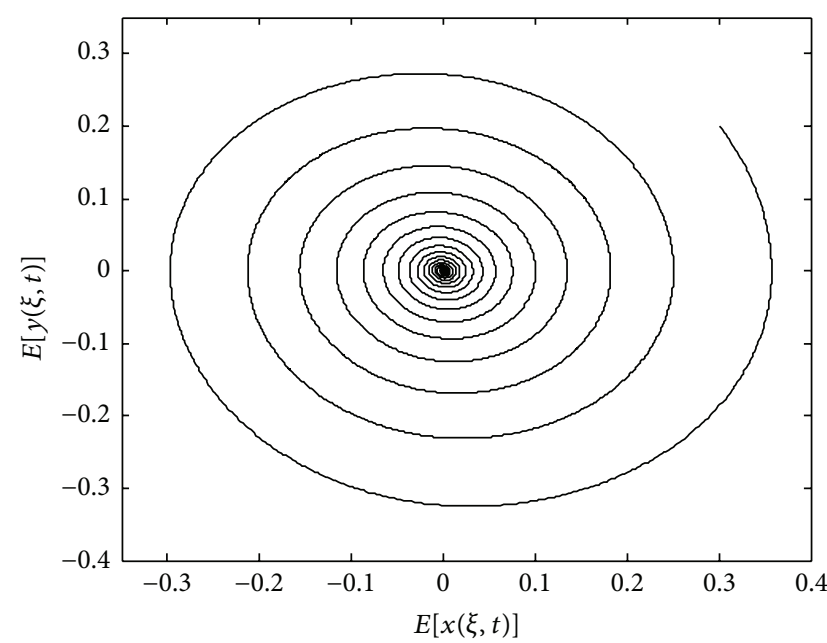

(a) $\sigma=0.1$ and $\beta<\beta_{c}$

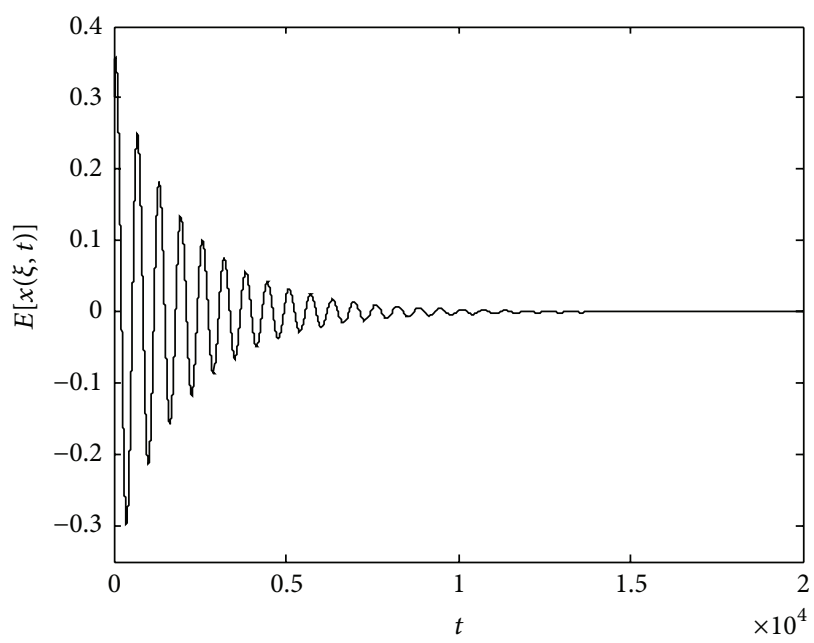

(c) $\sigma=0.1$ and $\beta<\beta_{c}$

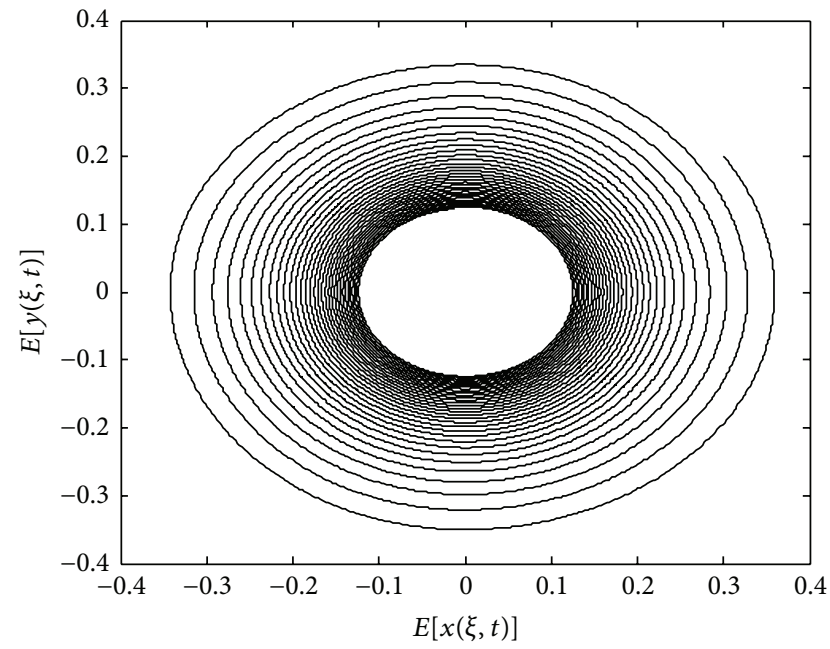

(b) $\sigma=0.1$ and $\beta>\beta_{c}$

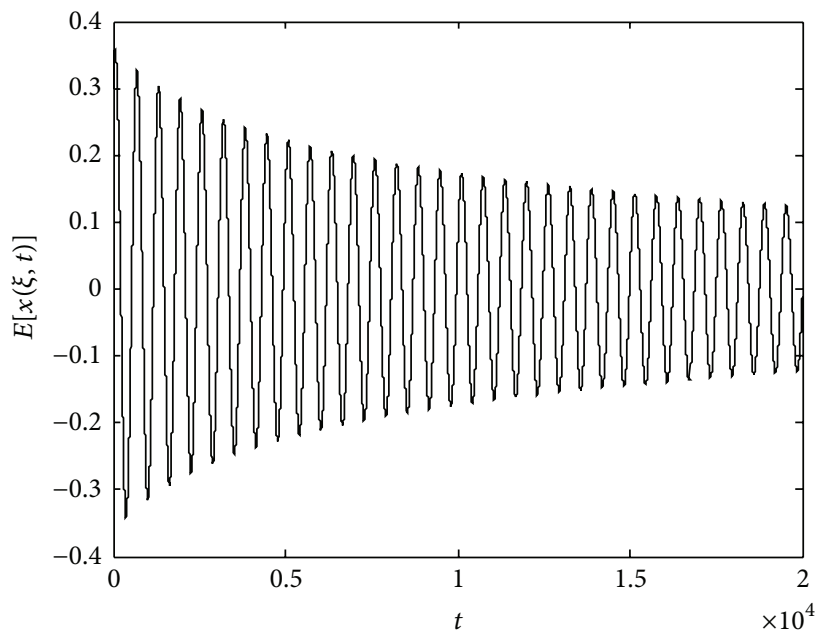

(d) $\sigma=0.1$ and $\beta>\beta_{c}$

FIGURE 4: The bifurcation phase diagrams and time history diagrams of van der Pol system with Gaussian white noise.

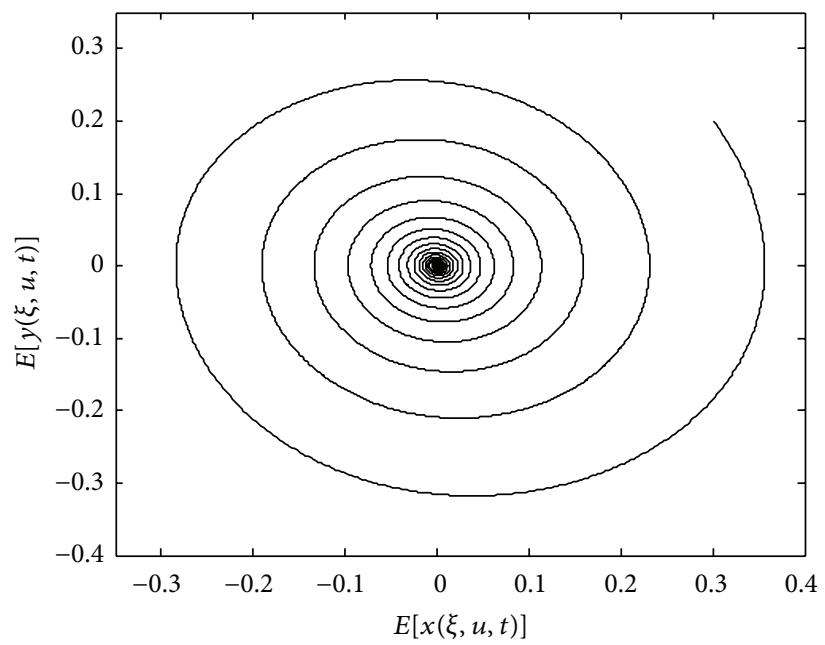

(a) $\delta=\sigma=0.1$ and $\beta<\beta_{c}$

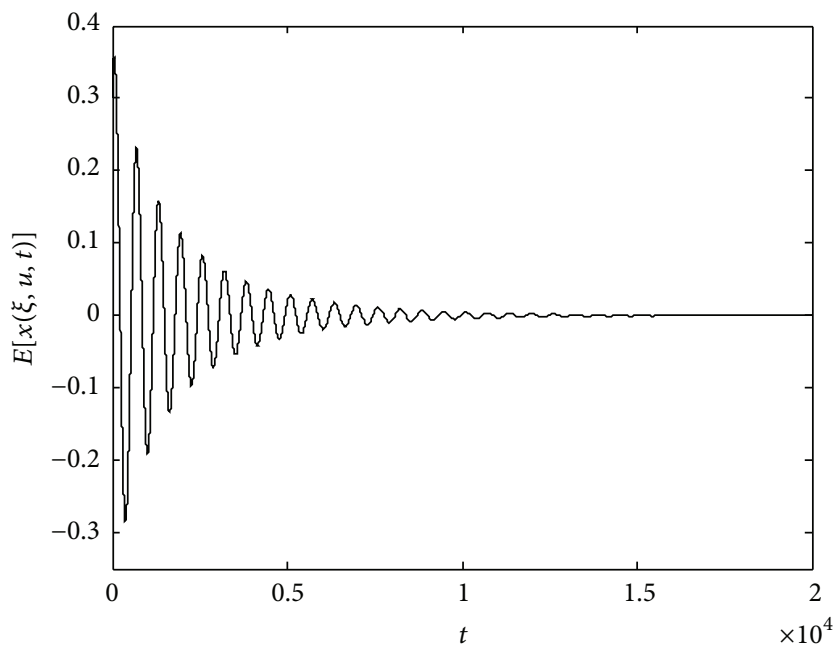

(b) $\delta=\sigma=0.1$ and $\beta<\beta_{c}$

FIgURE 5: The bifurcation phase diagrams and time history diagrams of compound stochastic van der Pol system. 


\section{Acknowledgments}

This work is supported by the National Natural Science Foundation of China (Grant no. 11362001).

\section{References}

[1] G. E. Young and R. J. Chang, "Prediction of the response of non-linear oscillators under stochastic parametric and external excitations," International Journal of Non-Linear Mechanics, vol. 22, no. 2, pp. 151-160, 1987.

[2] W. Q. Zhu and J. S. Yu, "The response of Van der Pol oscillator to Gaussian white noise," Journal of Vibration Engineering, vol. 1, no. 1, pp. 14-25, 1987.

[3] Y. Hao and Z. Q. Wu, "Stochastic P-bifurcation of tri-stable van der pol-duffing oscillator," Chinese Journal of Theoretical and Applied Mechanics, vol. 45, no. 2, pp. 257-264, 2013.

[4] Y. S. Chen and Q. J. Cao, "The Hopf bifurcation of the Van der Pol-Duffing oscillator in the presence of both parametric and the external influence," Acta Mechanica Sinica, vol. 25, no. 4, pp. 411-418, 1993.

[5] H. Yang, H. Zhu, and Y. Q. Chu, "Hopf bifurcation of Van der Pol oscillator under stochastic excitations," Journal of Beijing Institute of Technology, no. 13, pp. 279-285, 1993.

[6] L. Zhou and F. Chen, "Chaotic motions of the duffing-van der pol oscillator with external and parametric excitations," Shock and Vibration, vol. 2014, Article ID 131637, 5 pages, 2014.

[7] H. K. Leung, "Stochastic Hopf bifurcation in a biased van der Pol model," Physica A: Statistical Mechanics and Its Applications, vol. 254, no. 1-2, pp. 146-155, 1998.

[8] I. Opris and N. Rimbu, "Bifurcation phenomena of the delay correlated noise driven Van der Pol oscillator," General Physics, vol. 40, no. 1, pp. 3-10, 1994.

[9] W. Q. Zhu and J. S. Yu, “The response of Van der Pol oscillator to Gaussian white noise," Journal of Vibration Engineering, vol. 1, no. 1, pp. 14-24, 1987.

[10] N. D. Anh, V. L. Zakovorotny, and D. N. Hao, "Response analysis of Van der Pol oscillator subjected to harmonic and random excitations," Probabilistic Engineering Mechanics, vol. 37, pp. 5159, 2014.

[11] W. D. Iwan and P. T. Spanos, "Response envelope statistics for nonlinear oscillators with random excitation," Journal of Applied Mechanics, vol. 45, no. 1, pp. 170-174, 1978.

[12] W. F. Wu and Y. K. Lin, "Cumulant-neglect closure for nonlinear oscillators under random parametric and external excitations," International Journal of Non-Linear Mechanics, vol. 19, no. 4, pp. 349-362, 1984.

[13] W.-Y. Liu, W.-Q. Zhu, Z.-L. Huang, and Z.-L. Xiao, "Chaotic motion of Duffing oscillator under parametric excitation of bounded noise," Engineering Mechanics, vol. 16, no. 6, pp. 133$136,1999$.

[14] J. Zhang and B. Ellingwood, "Orthogonal series expansions of random fields in reliability analysis," Journal of Engineering Mechanics, vol. 120, no. 12, pp. 2660-2677, 1994.

[15] I. Braud and Ch. Obled, "On the use of Empirical Orthogonal Function (EOF) analysis in the simulation of random fields," Stochastic Hydrology and Hydraulics, vol. 5, no. 2, pp. 125-134, 1991.

[16] H. Jensen and W. D. Iwan, "Response of systems with uncertain parameters to stochastic excitation," Journal of Engineering Mechanics, vol. 118, no. 5, pp. 1012-1025, 1992.
[17] H. Jensen and W. D. Iwan, "Response variability in structural dynamics," Earthquake Engineering \& Structural Dynamics, vol. 20, no. 10, pp. 949-959, 1991.

[18] J. Li, Z. J. Liu, and J. Chen, "Orthogonal expansion of ground motion and PDEM-based seismic response analysis of nonlinear structures," Earthquake Engineering \& Engineering Vibration, vol. 8, no. 3, pp. 313-328, 2009.

[19] T. Fang, X. L. Leng, and C. Q. Song, "Chebyshev polynomial approximation for dynamical response problem of random system," Journal of Sound and Vibration, vol. 266, no. 1, pp. 198206, 2003.

[20] T. Fang, X. Leng, X. Ma, and G. Meng, “ $\lambda$-PDF and gegenbauer polynomial approximation for dynamic response problems of random structures," Acta Mechanica Sinica, vol. 20, no. 3, pp. 292-298, 2004.

[21] X. P. Ma, X. L. Leng, G. Meng, and T. Fang, "Evolutionary earthquake response of uncertain structure with bounded random parameter," Probabilistic Engineering Mechanics, vol. 19, no. 3, pp. 239-246, 2004.

[22] S. J. Ma, W. Xu, W. Li, and T. Fang, "Analysis of stochastic bifurcation and chaos in stochastic Duffing-van der Pol system via Chebyshev polynomial approximation," Chinese Physics, vol. 15, no. 6, pp. 1009-1963, 2006.

[23] S.-J. Ma, D. Dong, and M.-S. Yang, "Stochastic Hopf bifurcation analysis in a stochastic lagged logistic discrete-time system with Poisson distribution coefficient," Nonlinear Dynamics, vol. 80, no. 1-2, pp. 269-279, 2015.

[24] Y. Xu, S. Ma, and H. Zhang, "Hopf bifurcation control for stochastic dynamical system with nonlinear random feedback method," Nonlinear Dynamics, vol. 65, no. 1, pp. 77-84, 2011.

[25] S.-J. Ma, "Hopf bifurcation in a kind of stochastic van der Pol system," Acta Physica Sinica, vol. 60, no. 1, Article ID 010502, 2011.

[26] Y. M. Zhang and X. F. Zhang, "Reliability analysis of a double random Duffing system," Acta Physica Sinica, vol. 57, no. 7, pp. 3989-3995, 2008.

[27] P. Xiang, Y. Zhao, and J.-H. Lin, "Hybrid PC-PEM for complex random vibration analysis," Journal of Vibration and Shock, vol. 34, no. 4, pp. 35-39, 2015.

[28] J. Li and J. B. Chen, Stochastic Dynamics of Structures, John Wiley \& Sons (Asia), Singapore, 2009.

[29] J. Li, "The expanded order system method of combined random vibration analysis," Acta Mechanica Sinica, vol. 28, no. 1, pp. 6675, 1996.

[30] J. Liao, X.-R. Kong, Z.-X. Yang, and D.-F. Xu, “The orthogonal method for random structural non-stationary response," Engineering Mechanics, vol. 28, no. 12, pp. 13-19, 2011.

[31] K. Karhunen, "Uber lineare methoden in der wahrscheinlichkeitsrechnung," Annales Academiae Scientiarum Fennicae Series A: Mathematic-Physica, vol. 37, pp. 1-79, 1947.

[32] M. Loeve, Probability Theory, Springer, Berlin, Germany, 1997.

[33] S. P. Huang, S. T. Quek, and K. K. Phoon, "Convergence study of the truncated Karhunen-Loeve expansion for simulation of stochastic processes," International Journal for Numerical Methods in Engineering, vol. 52, no. 9, pp. 1029-1043, 2001.

[34] R. G. Jaimez and M. J. V. Bonnet, "On the Karhunen-Loeve expansion for transformed processes," Trabajos de Estadistica, vol. 2, no. 2, pp. 81-90, 1987.

[35] J. Guckenheimer and P. J. Holmes, Nonlinear Oscillations, Dynamical Systems, and Bifurcations of Vector Fields, Springer, New York, NY, USA, 1983.

[36] B. Hassard, N. Kazarinoff, and Y.-H. Wan, Theory and Applications of Hopf Bifurcation, Cambridge University Press, 1981. 


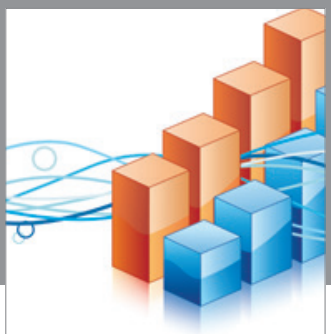

Advances in

Operations Research

vatem alat4

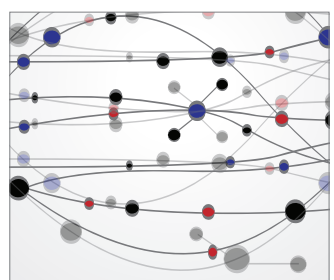

\section{The Scientific} World Journal
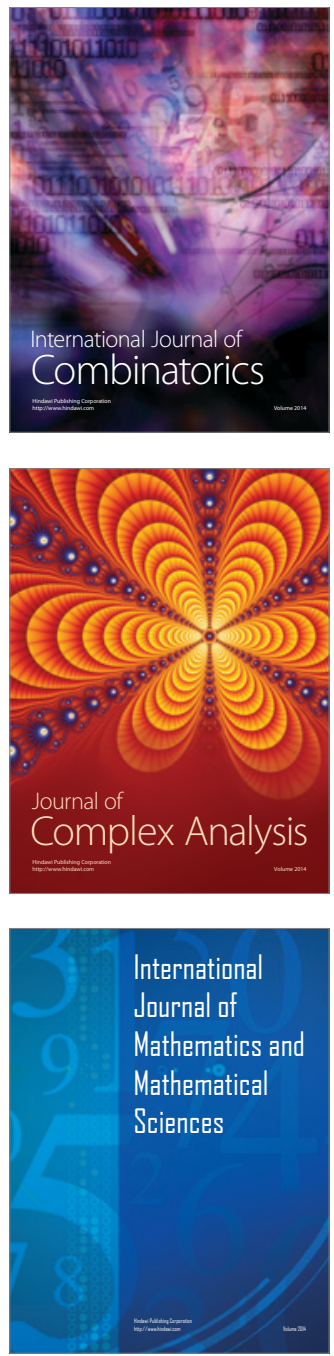
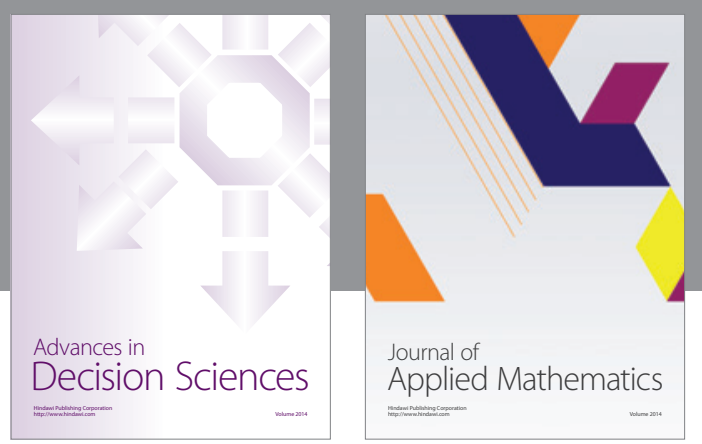

Algebra

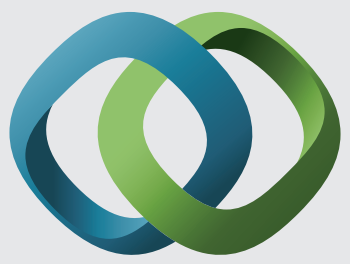

\section{Hindawi}

Submit your manuscripts at

http://www.hindawi.com
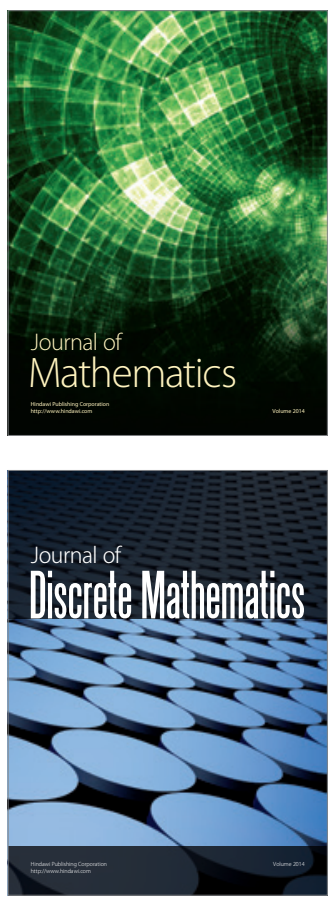

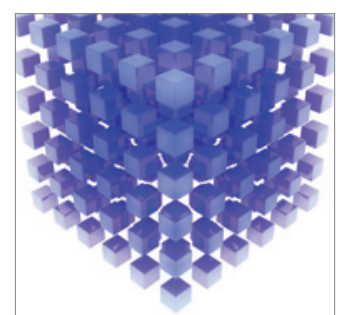

Mathematical Problems in Engineering
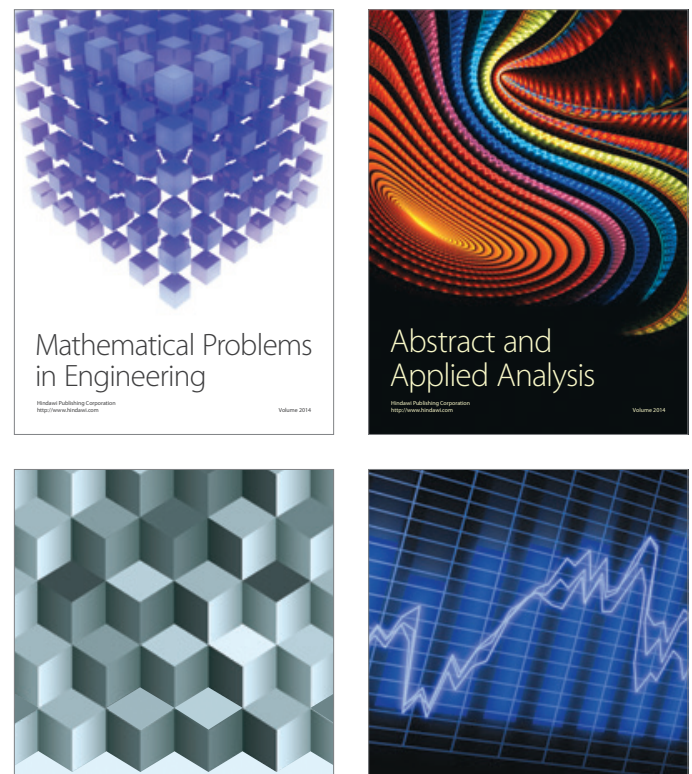

Journal of

Function Spaces

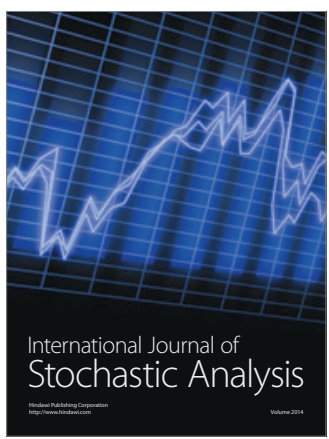

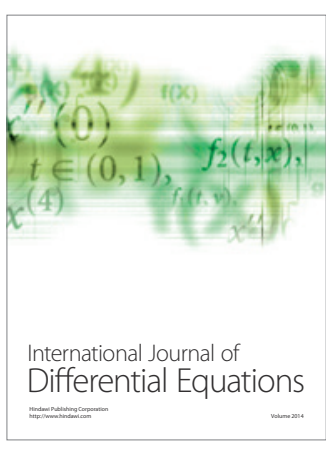
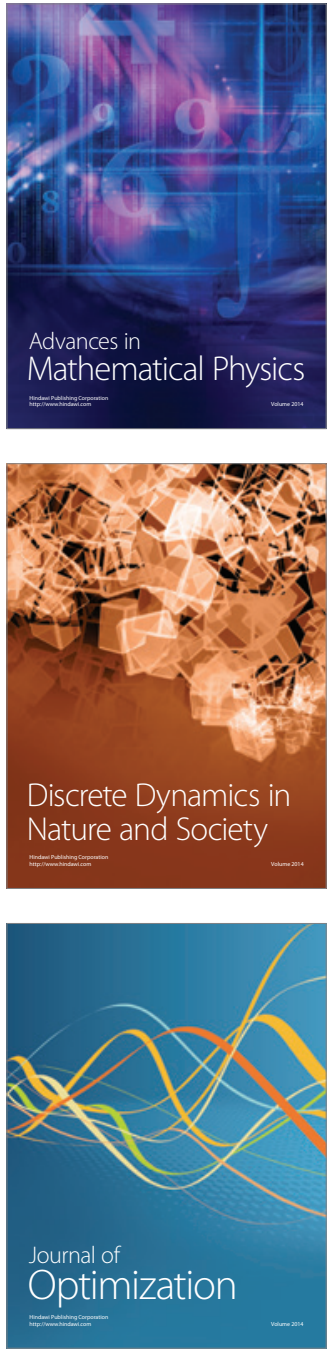